\title{
Role of extracytoplasmic function sigma factors in biofilm formation of Porphyromonas gingivalis
}

Satosu Onozawa ${ }^{1 \dagger}$, Yuichiro Kikuchii ${ }^{2,{ }^{*}+}$, Kazuko Shibayama ${ }^{3}$, Eitoyo Kokubu²,3, Masaaki Nakayama ${ }^{4}$, Tetsuyoshi Inoue ${ }^{4}$, Keisuke Nakano ${ }^{5,6}$, Yukinaga Shibata ${ }^{1}$, Naoya Ohara ${ }^{4}$, Koji Nakayama ${ }^{7}$, Kazuyuki Ishihara ${ }^{3}$, Toshiyuki Kawakami ${ }^{6}$ and Hiromasa Hasegawa ${ }^{5,6}$

\begin{abstract}
Background: Porphyromonas gingivalis has been implicated as a major pathogen in the development and progression of chronic periodontitis. P. gingivalis biofilm formation in the subgingival crevice plays an important role in the ability of the bacteria to tolerate stress signals outside the cytoplasmic membrane. Some bacteria use a distinct subfamily of sigma factors to regulate their extracytoplasmic functions (the ECF subfamily). The objective of this study was to determine if $P$. gingivalis ECF sigma factors affect $P$. gingivalis biofilm formation.

Methods: To elucidate the role of ECF sigma factors in P. gingivalis, chromosomal mutants carrying a disruption of each ECF sigma factor-encoding gene were constructed. Bacterial growth curves were measured by determining the turbidity of bacterial cultures. The quantity of biofilm growing on plates was evaluated by crystal violet staining.

Results: Comparison of the growth curves of wild-type $P$. gingivalis strain 33277 and the ECF mutants indicated that the growth rate of the mutants was slightly lower than that of the wild-type strain. The PGN_0274- and PGN_1740-defective mutants had increased biofilm formation compared with the wild-type $(p<0.001)$; however, the other ECF sigma factor mutants or the complemented strains did not enhance biofilm formation.

Conclusion: These results suggest that PGN_0274 and PGN_1740 play a key role in biofilm formation by $P$. gingivalis.

Keywords: Extracytoplasmic function sigma factor, Biofilm, Porphyromonas gingivalis, Periodontal disease
\end{abstract}

\section{Background}

The anaerobic Gram-negative bacterium Porphyromonas gingivalis is considered one of the important etiological agents of periodontal disease [1]. To colonize and survive in the gingival crevice, $P$. gingivalis must be capable of sensing and responding to the prevailing environmental conditions, including variations in temperature, oxygen tension, $\mathrm{pH}$, nutrient availability and the presence of other bacterial cells. P. gingivalis possesses transcriptional regulators that have been implicated in protection against heat shock stress or oxidative stress, such as RprY $[2,3]$ and OxyR [4]. In addition, this bacterium and other bacteria form biofilms to protect against environmental

\footnotetext{
* Correspondence: yukikuchi@tdc.ac.jp

${ }^{\dagger}$ Equal contributors

${ }^{2}$ Oral Health Science Center, Tokyo Dental College, 2-9-18, Misaki-cho,

Chiyoda-ku, Tokyo, Japan

${ }^{3}$ Department of Microbiology, Tokyo Dental College, 2-1-14, Misaki-cho,

Chiyoda-ku, Tokyo, Japan

Full list of author information is available at the end of the article
}

stress [5]. Dental plaque, a multispecies biofilm, is organized on the tooth surface and periodontal tissues of the human oral cavity [6]. Oral bacteria in the biofilms survive in the gingival crevice for a long period of time, leading to gingivitis that can eventually progress into periodontitis. Understanding how bacteria escape environmental stress is very important for the prevention of periodontal disease.

Extracytoplasmic function (ECF) sigma factors serve as bacterial transcriptional regulators in the response to various stresses. The wild-type $P$. gingivalis 33277 genome encodes six ECF sigma factors (PGN_0274, PGN_0319, PGN_0450, PGN_0970, PGN_1108 and PGN_1740; GenBank: AP009380) [7]. PGN_1108 (W83 ORF number: PG1318) plays a role in the regulation of mutation frequency in the bacterium [8]. PGN_0274 (W83 ORF number: PG0162) and PGN_0450 (W83 ORF number: PG1660) may be involved in the post-transcriptional regulation of gingipain [9], and PGN_1740 (W83 ORF 
number: PG1827) is required for survival of the bacterium in the presence of oxygen and oxidative stress, hemin uptake and virulence $[9,10]$.

In this study, to analyze the role of ECF sigma factors in $P$. gingivalis biofilm formation, disruption of the ECF sigma factors, except PGN_1108, was performed. The PGN 1108-defective mutant may have a mutator phenotype, and we therefore excluded it from our experiments in this study [8]. The PGN_0274 and PGN_1740-defective mutants exhibited enhanced biofilm formation, but the complemented strains did not. These results suggest that the PGN_0274 and PGN_1740 ECF sigma factors are involved in the regulation of biofilm formation in the bacterium.

\section{Methods}

\section{Bacterial strains and cell culture conditions}

All bacterial strains and plasmids used in the present study are listed in Table 1. P. gingivalis cells were grown anaerobically $\left(10 \% \mathrm{CO}_{2}, 10 \% \mathrm{H}_{2}\right.$ and $\left.80 \% \mathrm{~N}_{2}\right)$ in enriched brain heart infusion (BHI) broth and on enriched tryptic soy (TS) agar [11]. For blood agar plates, defibrinated laked sheep blood was added to enriched TS agar at $5 \%$. For selection and maintenance of antibiotic-resistant $P$. gingivalis strains, the following antibiotics were added to the medium: $15 \mu \mathrm{g} / \mathrm{ml}$ erythromycin (Em), and $0.7 \mu \mathrm{g} / \mathrm{ml}$ tetracycline (Tc).

\section{Construction of ECF sigma factor mutants and complemented mutant strains}

To disrupt the ECF sigma factor genes, PGN_0274-, PGN_0319-, PGN_0450-, PGN_0970- and PGN_1740encoding genes were PCR-amplified from the chromosomal DNA of $P$. gingivalis 33277 using Takara Ex Taq (Takara Bio, Otsu, Japan) and the gene-specific primers listed in Table 2. The amplified areas were a DNA fragment containing part of the $5^{\prime}$ end of each ECF sigma

Table 1 Bacterial strains and plasmids used in this study

\begin{tabular}{|c|c|c|}
\hline Strain or plasmid & Description & Reference or source \\
\hline \multicolumn{3}{|l|}{ E. coli strain } \\
\hline $\mathrm{DH} 5 \mathrm{a}$ & General-purpose host strain for cloning & Invitrogen \\
\hline \multicolumn{3}{|l|}{ P. gingivalis strain } \\
\hline 33277 & wild type & ATCC \\
\hline KDP314 & PGN_0274::ermF ermAM, Em ${ }^{r}$ & This study \\
\hline KDP315 & PGN_0319::ermF ermAM, Em ${ }^{r}$ & This study \\
\hline KDP316 & PGN_0450::ermF ermAM, Em ${ }^{r}$ & This study \\
\hline KDP317 & PGN_0970::ermF ermAM, Em ${ }^{r}$ & This study \\
\hline KDP319 & PGN_1740::ermF ermAM, Em ${ }^{r}$ & This study \\
\hline KDP314C & KDP314/pKD828, Em ${ }^{r} \mathrm{TC}^{\mathrm{r}}$ & This study \\
\hline KDP319C & KDP319/pKD829, Em ${ }^{r} \mathrm{Tc}^{r}$ & This study \\
\hline \multicolumn{3}{|l|}{ E. coli plasmid } \\
\hline pGEM-T Easy & Ap ${ }^{r}$, plasmid vector for TA cloning & Promega \\
\hline pKD355 & $A p^{r}$, contains the ermF ermAM DNA cassette between EcoRI and BamHI of pUC18 & 12 \\
\hline pKD814 & Ap ${ }^{r}$, contains the 1.0-kb PCR-amplified fragment (PGN_0450 region) in pGEM-T Easy & This study \\
\hline pKD817 & Ap ${ }^{r}$, contains the 1.5-kb PCR-amplified fragment (PGN_0274 region) in pGEM-T Easy & This study \\
\hline pKD818 & Ap ${ }^{r}$, contains the 2.0-kb PCR-amplified fragment (PGN_0319 region) in pGEM-T Easy & This study \\
\hline pKD819 & Ap ${ }^{r}$, contains the 2.0-kb PCR-amplified fragment (PGN_0970 region) in pGEM-T Easy & This study \\
\hline pKD821 & Ap ${ }^{r}$, contains the 2.0-kb PCR-amplified fragment (PGN_1740 region) in pGEM-T Easy & This study \\
\hline pKD822 & $\mathrm{Ap}^{r} \mathrm{Em}^{r}$, contains the ermF ermAM DNA cassette at BamHI site within PGN_0274 of pKD817 & This study \\
\hline pKD823 & $\mathrm{Ap}^{r} \mathrm{Em}^{r}$, contains the ermF ermAM DNA cassette at BamHI site within PGN_0319 of pKD818 & This study \\
\hline pKD824 & $\mathrm{Ap}^{r} \mathrm{Em}^{\mathrm{r}}$, contains the ermF ermAM DNA cassette at BamHI site within PGN_0450 of pKD814 & This study \\
\hline pKD825 & $\mathrm{Ap}^{\mathrm{r}} \mathrm{Em}^{\mathrm{r}}$, contains the ermF ermAM DNA cassette at Bg/ll site within PGN_0970 of pKD819 & This study \\
\hline pKD827 & $\mathrm{Ap}^{r} \mathrm{Em}^{r}$, contains the ermF ermAM DNA cassette at BamHI site within PGN_1740 of pKD821 & This study \\
\hline \multicolumn{3}{|l|}{ P. gingivalis plasmid } \\
\hline pT-COW & $A p^{r} T C^{r}, E$. coli-P. gingivalis shuttle plasmid & 14 \\
\hline pKD828 & Ap ${ }^{r} T c^{r}$, pT-COW-PGN_0274 & This study \\
\hline pKD829 & Ap ${ }^{r} T c^{r}$, pT-COW-PGN_1740 & This study \\
\hline
\end{tabular}


Table 2 Primers used in this study

\begin{tabular}{ll}
\hline Name & Nucleotide sequence (5'-3') \\
\hline PGN0274-U-F & TCGACAGTTGATGCCGAT \\
PGN0274-U-R-BamHI & GGGATCCCCATCGAAAGACTGCAATCTGG \\
PGN0274-D-F-BamHI & GGGATCCCATGACGACGCCGCTCCTGTCGAAA \\
PGN0274-D-R & TGTGCAAAAAAGGAACAGC \\
PGN0319-U-F & GCTGCCGCTCCTCTTCAT \\
PGN0319-U-R-BamHI & GGGATCCCAAAGGCAGATCGTCCGGTA \\
PGN0319-D-F-BamHI & GGGATCCCCTCCGATCATGCCCCTA \\
PGN0319-D-R & TCAGGCTCTTGTACAGATGGA \\
PGN0450-U-F & GGGATGTGGAGAAAAAGGAA \\
PGN0450-D-R & ATGACCACGGACAGGAAGAT \\
PGN0970-U-F & ACCGGGAATAATTCTCAAGC \\
PGN0970-U-R-Bgll & AAGATCTTCCAAAGAGGTCGGATAAGGA \\
PGN0970-D-F- Bg/I & AAGATCTTAGGCTGCCGAGGTACAGGA \\
PGN0970-D-R & ACACAAGCTACAGCCCCGTA \\
PGN1740-U-F & GAGGATCTCCCTGCCAATAAT \\
PGN1740-U-R-BamHI & GGGATCCCACCCAGCCTTGAAGTGACA \\
PGN1740-D-F-BamHI & GGGATCCCGCTCACTGTCATGCGAAAT \\
PGN1740-D-R & CCAACGGCTATTAGCATCC \\
PGN0274-COMP-U-F-PstI & CCTGCAGGCTGCTACTGTCTCGGACGTG \\
PGN0274-COMP-D-R- & GGGATCCCGTTGGGTTGAGGCTGCAT \\
BamHI & \\
PGN1740-COMP-U-F- & CGGGATCCCGAGTGCGATATCGGGAATCAG \\
BamHI & \\
PGN1740-COMP-D-R- & CGGGATCCCGAGTTGATACGGCTGCTATGC \\
BamHI & \\
\hline Restriction sites incorporated into oligonucleotides for subcloning are bold.
\end{tabular}

Restriction sites incorporated into oligonucleotides for subcloning are bold.

factor gene and the upstream region of the ATG initiation codon, and a DNA fragment containing the 3' end of each sigma factor gene and the downstream region of its stop codon. Both fragments were then ligated into the multiple cloning site of T-vector (pGEM-T Easy Vector, Promega, Tokyo, Japan). A BamHI-SacI fragment (BglII-SacI fragment for PGN_0970) containing the $3^{\prime}$ end of each sigma factor gene was extracted from the resulting plasmid and ligated into the BamHI-SacI site (BglII-SacI fragment for PGN_0970) of the plasmid containing the $5^{\prime}$ end of the corresponding ECF gene. The ermF-ermAM cassette of pKD355 [12] was inserted into the BamHI site within PGN_0274 of pKD817, PGN_0319 of pKD818, PGN_0450 of pKD814 and PGN_1740 of pKD821, or the BglII site within PGN_0970 of pKD819 to yield pKD822, pKD823, pKD824, pKD827 and pKD825, respectively. These plasmids were linearized by NotI digestion and introduced into $P$. gingivalis 33277 cells by electroporation as described previously [13], resulting in KDP314 (PGN_0274::ermF ermAM),
KDP315 (PGN_0319:: ermF ermAM), KDP316 (PGN_0450:: ermF ermAM), KDP317 (PGN_0970:: ermF ermAM) and KDP319 (PGN_1740:: ermF ermAM). Correct gene replacement of these strains, which had been generated by double crossover recombination events, was verified by PCR and Southern blot analysis (data not shown).

For complementation of PGN_0274 and PGN_1740, the whole ECF sigma factor gene region with its upstream and downstream flanking regions $(0.5 \mathrm{~kb})$ was PCRamplified from the chromosomal DNA using Takara Ex Taq with the upper and lower primers (Table 2). The amplified DNA fragments were ligated into the multiple cloning site of pGEM T-Easy vector. The SphI-BamHI fragment of PGN_0274 or the BamHI fragment of PGN_1740 were extracted from the resulting plasmid and ligated into the SphI-BamHI or BamHI site of pT-COW [14], which was kindly provided by Professor N. B. Shoemaker (University of Illinois at UrbanaChampaign, USA). The resulting plasmids, pKD828 and pKD829, were introduced into KDP314 or KDP319 by electroporation, resulting in KDP314C and KDP319C, respectively, after $7 \mathrm{~d}$ incubation on enriched TS agar containing $0.7 \mu \mathrm{g} / \mathrm{ml}$ tetracycline. The presence of pT-COW-derived plasmid was verified by PCR and restoration of the mRNA of the mutated gene was established by RT-PCR (data not shown).

\section{Evaluation of biofilm formation ability}

Biofilm formation was examined by the modified protocol of Saito [15]. In brief, $P$. gingivalis cells were inoculated into $\mathrm{BHI}$ broth, and precultured anaerobically at $37^{\circ} \mathrm{C}$ for 2 d. Fully grown cultures of the $P$. gingivalis strains had turbidity adjusted to $\mathrm{OD}_{660}=0.1$ with fresh medium, and then 1.5-ml aliquots were inoculated into collagen type-Icoated 12-well flat-bottom microplates (IWAKI Glass Co., Funabashi, Japan) and cultured anaerobically at $37^{\circ} \mathrm{C}$ for $2 \mathrm{~d}$. The culture medium was then removed from each well and $0.5 \mathrm{ml}$ of $0.1 \%$ crystal violet solution was added. After $15 \mathrm{~min}$, the wells were rinsed three times with PBS and air-dried. The crystal violet remaining in the biofilm was solubilized with $0.5 \mathrm{ml}$ of $1 \%$ SDS and absorbance was measured at $\mathrm{A}_{600}$ using a microplate reader (Molecular Devices, Sunnyvale, CA, USA). Biofilm mass was determined by crystal violet staining and adjusted for growth ( $\mathrm{A}_{600}$ units per $\mathrm{OD}_{660}$ unit).

\section{Statistical analysis}

The one-way ANOVA Test/Dunnett's Multiple Comparison Test was used to compare the differences between 33277 and ECF mutants using GraphPad Prism version 6.0 for Windows (GraphPad Software, Inc., La Jolla, CA, USA). Data were considered significant if $p<0.05$. 


\section{Results}

Growth and biofilm formation ability of the $P$. gingivalis ECF sigma factor mutants

All five ECF mutants grew more slowly than the wildtype strain in exponential phase, and final yields of the ECF mutants were less than that of the wild-type following a 48-h incubation under anaerobic conditions (Figure 1). The PGN_1740 mutant showed remarkably slow growth compared with wild-type and other ECF mutants. To evaluate the relationship between the biofilm formation activity and ECF sigma factors, the biofilm formation was examined for the wild-type and five ECF mutants. After crystal violet staining of the biofilm, it was first solubilized with ethanol. The crystal violet remaining in the wild-type, PGN_0319, PGN_0450, PGN_0970 and PGN_1740 mutant biofilm was solubilized and extracted, but in the PGN_0274 mutant, solubilization and extraction were not complete (see Additional file 1). Thus, the biofilm mass of all tested strains was dissolved with SDS and measured. Among the ECF mutants, the biofilm mass of the PGN_0274 and PGN_1740 mutants was higher than the wild-type (Figure 2). To confirm collagen type-I influences biofilm formation, we investigated the biofilm formation using a non-coated plate. The results were almost the same (see Additional file 2), as the PGN_0274 mutant produced more biofilm than the wild-type. However, the wild-type strain and the PGN_1740 mutant were not statistically different. This result suggested the biofilm formation by the PGN_1740 mutant was influenced by the environmental situation, such as the presence of collagen type-I.

\section{Complementation of the PGN_0274- and PGN_1740-defective mutants}

To determine if the enhanced biofilm mass was caused by the deletion of PGN_0274 and PGN_1740, we constructed strains where the PGN_0274 and PGN_1740 were restored. The PGN_0274 and PGN_1740 complemented strains were constructed by introduction of the pT-COW containing the wild-type PGN_0274 and PGN_1740 into each of the mutants. This complementation restored the biofilm formation ability to the wild-type levels (Figure 3). These results support the concept that PGN_0274 and PGN_1740 play an important role in controlling $P$. gingivalis biofilm formation.

\section{Discussion}

Bacteria sometimes encounter an environment unfavorable to their survival. The human oral microbiota is also often influenced by various stresses; hence, it must possess the ability to defend itself. Two principal regulatory mechanisms interact with cytoplasmic and extracytoplasmic regions via alternative ECF sigma factors and phosphorylation-dependent response regulators (twocomponent systems, TCSs) $[16,17]$. ECF sigma factors have been shown to regulate cell envelope-related processes (involving maintenance of the membrane/periplasmic architecture), such as secretion, synthesis of exopolysaccharides, iron export and efflux synthesis of extracellular proteases [18]. Bacterial core RNA polymerase (composed of two $\alpha$ subunits, $\beta$ subunit and $\beta$ ' subunit) binds sigma factors. Multiple sigma factors are the bacterial transcription initiation factors that enable specific binding of RNA

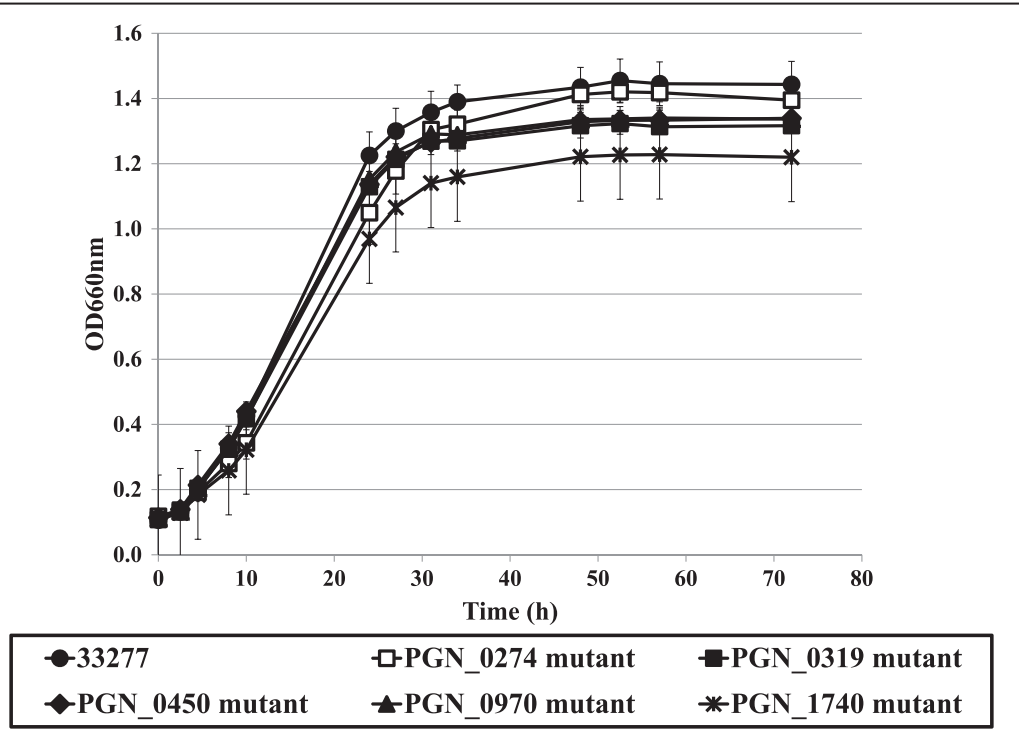

Figure 1 Growth of $\boldsymbol{P}$. gingivalis 33277 and ECF sigma factor mutants. Growth curves of P. gingivalis 33277 (wild-type; circle), PGN_0274 mutant (KDP314; open rectangle), PGN_0319 mutant (KDP315; closed rectangle), PGN_0450 mutant (KDP316; diamond), PGN_0970 mutant (KDP317; triangle), PGN_1740 mutant (KDP319; cross) in enriched BHI broth. The data shown are mean \pm SD of triplicate experiments. 


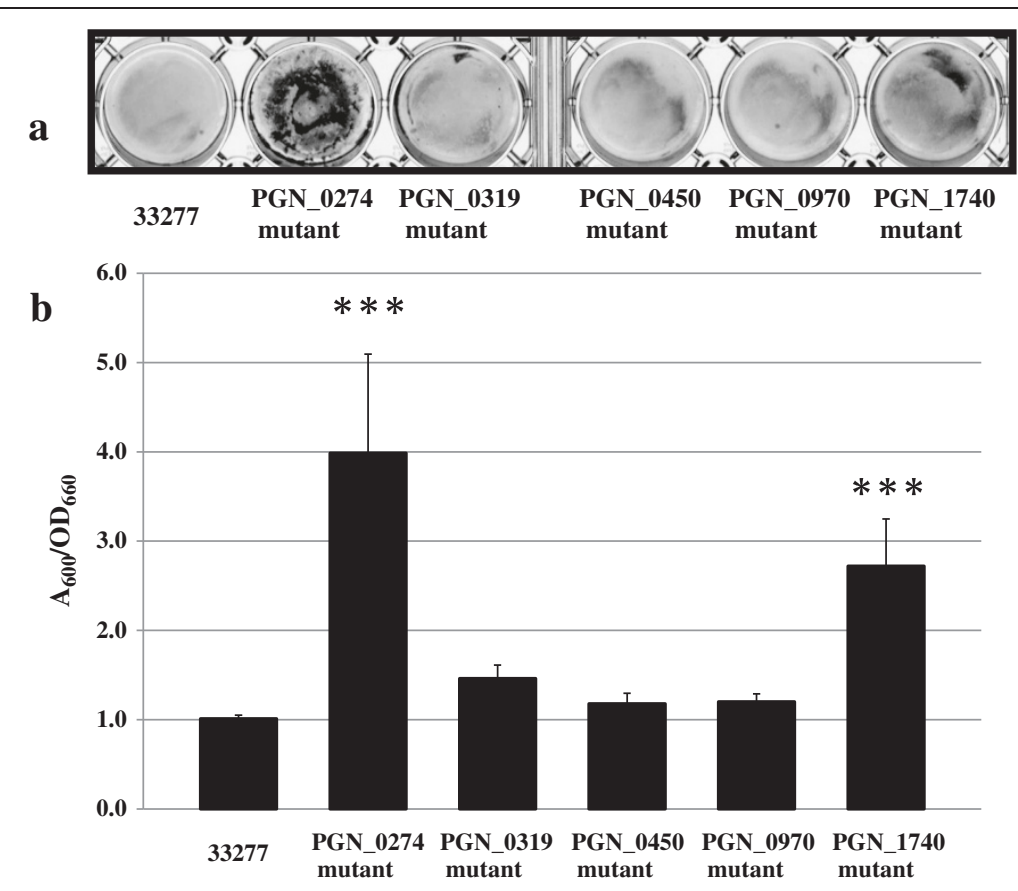

Figure 2 Biofilm formation by homotypic $P$. gingivalis 33277 or ECF sigma factor mutants. The strains were grown on enriched BHI broth anaerobically at $37^{\circ} \mathrm{C}$ in collagen type---coated 12 -well flat-bottom microplates. After $48 \mathrm{~h}$ of cultivation, the organized biofilm mass was evaluated by staining with crystal violet. (a) The photographs are a representative sample of each experimental strain. (b) Biofilm formation determined by crystal violet staining and adjusted for growth ( $\mathrm{A}_{600}$ units per $\mathrm{OD}_{660}$ unit). The data shown are mean $\pm \mathrm{SD}$ of triplicate experiments. ${ }^{* * *}, p<0.001$, by a one-way ANOVA Test/Dunnett's Multiple Comparison Test.

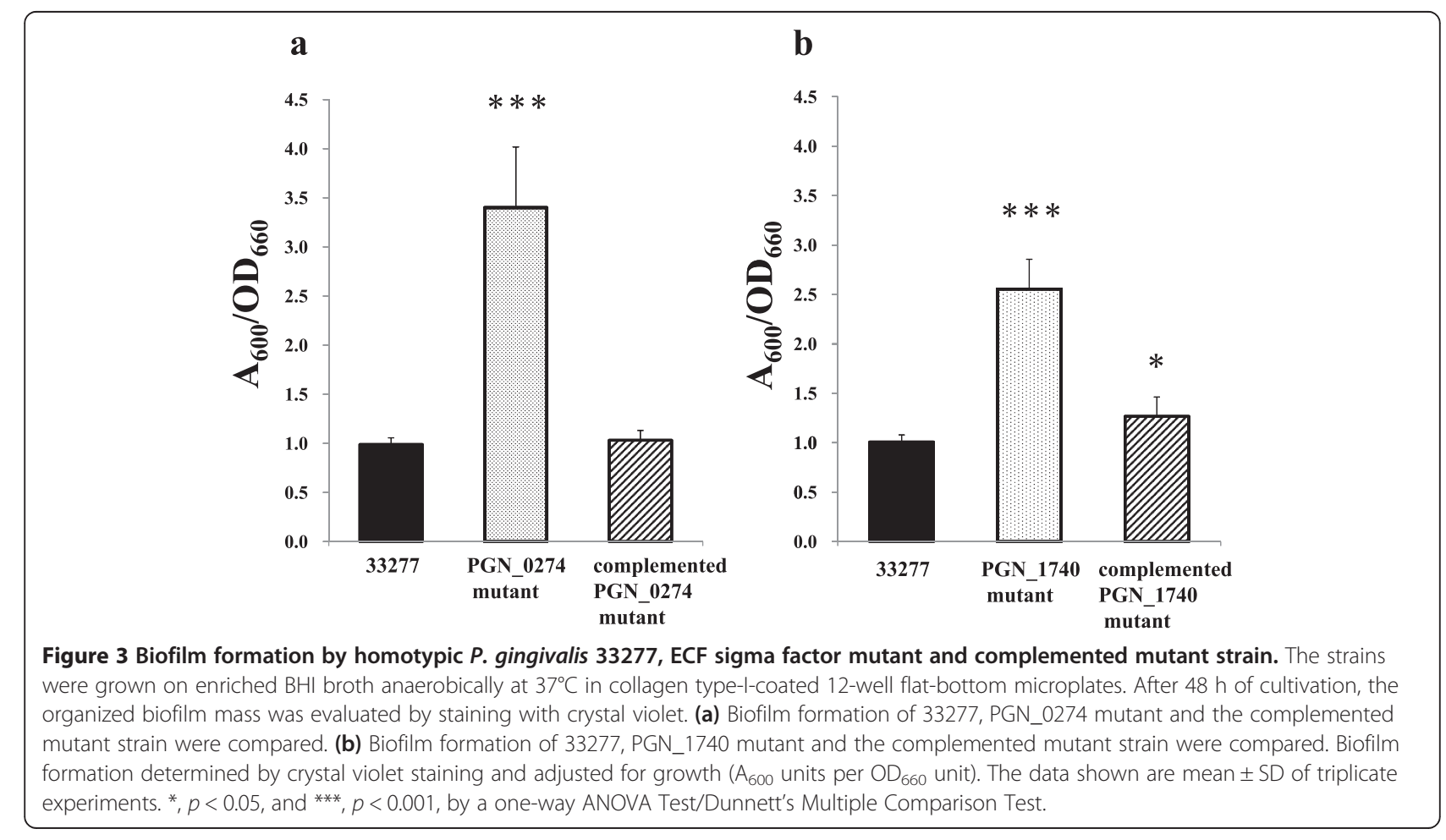


polymerase to gene promoters. In contrast, TCSs typically consist of a membrane-bound histidine kinase that senses a specific environmental stimulus and a corresponding response regulator that mediates the cellular response, mostly through differential expression of target genes [19]. Interestingly, a transcriptional regulator in Methylobacterium extorquens, PhyR, has been identified and determined to combine domains of both systems [20]. Taken together, ECF sigma factors and TCS are essential factors that protect bacteria from environmental stress.

Several $P$. gingivalis ECF sigma factors have been previously described. Nevertheless, there is no information on the ECF sigma factors that may operate in this bacterium in response to biofilm formation. In Bacillus subtilis and Pseudomonas aeruginosa, ECF sigma factors are involved in regulating biofilm development $[21,22]$. In this study, we investigated whether biofilm formation of $P$. gingivalis is regulated by ECF sigma factors. This study demonstrated that PGN_0274 and PGN_1740 mutants yielded higher biofilm formation than that obtained with the wild-type or the other ECF sigma factor mutants. The inactivation of PGN_1740 also increased the expression of fims at the transcriptional level [9]. Fimbriae and minor fimbriae influence monospecies biofilms [23]. The transcriptional level of fims was examined using RT-PCR, which showed the fimS expression was downregulated (see Additional file 3 ). The results showed Fims may not be involved in controlling biofilm formation. Further work is needed to clarify this point.

The biofilm assay revealed that ethanol did not completely dissolve the biofilm mass and extract the crystal violet stain for the PGN_0274 mutant biofilm (see Additional file 1). Therefore, we dissolved the biofilm mass with SDS and measured the resulting crystal violet present in the sample. The need for a more stringent solvent suggested that the biofilm matrix around the mutant is composed partly of a protein component. The biofilm extracellular polymeric substances (EPS), composed of exopolysaccharides, proteins, nucleic acids and lipids, play a role as a defense structure, protecting bacteria from the host immune system and antimicrobial therapy [24]. Protein is a major component of EPS [25]. As the metabolic pathways of the PGN_0274 mutant are changed by the loss of the PGN_0274 ECF sigma factor, the protein yields in the PGN_0274 mutant are more abundant than those in the wild-type and the other mutants. Therefore, we examined the protein profile of the ECF sigma mutants compared with the wild-type (see Additional file 4). The degradation of 75-k-250-k Da proteins were demonstrated in the wild-type, PGN_0319, PGN_0450, PGN_0970 and PGN_1740 mutants, but not in the PGN_0274 mutant. This alteration was not observed in the presence of the proteinase inhibitors TLCK and leupeptin. The protein profiles of the wild-type and ECF sigma mutants were almost identical. Taken together, these results suggest this apparent difference in solubility might be explained by the decrease of Kgp and Rgp activity in the PGN_0274 mutant [9]. Kgp suppresses biofilm formation and Rgp controls microcolony morphology [26]. The $\operatorname{sinR}$ ortholog PGN_0088, a transcriptional regulator, acts as a negative regulator of exopolysaccharide accumulation in wild-type P. gingivalis [27]. PGN_0274 may control different metabolic pathways than PGN_0088 and act as a negative regulator of protein accumulation.

In conclusion, we have identified that PGN_0274 and PGN_1740 play a key role in P. gingivalis biofilm formation. These results show for the first time that $P$. gingivalis ECF sigma factors are involved in biofilm formation. PGN_0274 is involved in the post-transcriptional regulation of gingipains [8]. Gingipain is a very important virulence factor in $P$. gingivalis, because gingipains destroy periodontal tissue, immunoglobulins and complement factors $[28,29]$. As PGN_1740 plays a significant role in oxidative stress responses in the bacterium $[8,9]$, the survival of the PGN_1740 mutant was reduced in the presence of host cells [9]. We also observed this in Ca922 cells (data not shown). Taken together, these results show that ECF sigma factors PGN_0274 and PGN_1740 are involved in the virulence of $P$. gingivalis. Further studies on the roles of the $P$. gingivalis ECF sigma factors, PGN_0274 and PGN_1740, will help us understand the ability of $P$. gingivalis to colonize and survive in the gingival crevice, and therefore act as a human pathogen.

\section{Conclusions}

The mass of the biofilm of the PGN_0274 and PGN_1740 mutants was higher than that in the wild-type, suggesting P. gingivalis extracytoplasmic function sigma factors PGN_0274 and PGN_1740 are involved in biofilm formation.

\section{Additional files}

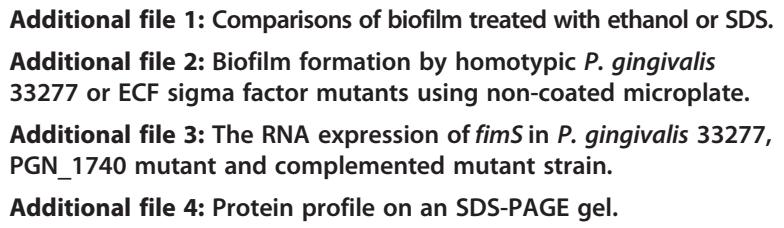

\section{Abbreviations}

ECF: Extracytoplasmic function; BHI: Brain heart infusion; TS: Tryptic soy; Em: Erythromycin; Tc: Tetracycline; PCR: Polymerase chain reaction; ANOVA: Analysis of variance; TCS: Two-component systems; SDS: Sodium dodecyl sulfate; EPS: Extracellular polymeric substances.

\section{Competing interests}

The authors declare that they have no competing interests. 


\section{Authors' contributions}

SO and YK contributed equally to this work. SO and YK planned the study, performed the experiments and data analysis, and wrote the manuscript. Koji Nakayama, $\mathrm{NO}$ and $\mathrm{Kl}$ participated in planning and designing the study as well as in the data analysis. MN and TI performed the survival study in host cells and helped to draft the manuscript. KS, EK and YS helped with the microbiology studies. Keisuke Nakano, TK and HH helped with the microbiology studies and supervised writing of the manuscript. All authors have read and approved the final manuscript.

\section{Acknowledgements}

We thank the members of the Department of Oral Microbiology, Matsumoto Dental University, and Microbiology, Tokyo Dental College, for helpful discussion. This study was supported by a Grant-in-Aid (24792372) for scientific research from the Ministry of Education, Science, Sports, Culture and Technology, Japan, And this research was also supported by Oral Health Science Center Grant hrc8 from Tokyo Dental College, and by a Project for Private Universities: matching fund subsidy from MEXT (Ministry of Education, Culture, Sports, Science and Technology) of Japan, 2010-2012.

\begin{abstract}
Author details
${ }^{1}$ Department of Oral Microbiology, Matsumoto Dental University, 1780 Gobara, Hiro-oka, Shiojiri, Japan. ${ }^{2}$ Oral Health Science Center, Tokyo Dental College, 2-9-18, Misaki-cho, Chiyoda-ku, Tokyo, Japan. ${ }^{3}$ Department of Microbiology, Tokyo Dental College, 2-1-14, Misaki-cho, Chiyoda-ku, Tokyo, Japan. ${ }^{4}$ Department of Oral Microbiology, Graduate School of Medicine, Dentistry and Pharmaceutical Sciences, Okayama University, 2-5-1 Shikata-cho, Kita-ku, Okayama, Japan. ${ }^{5}$ Department of Oral Pathology, Matsumoto Dental University School of Dentistry, 1780, Hirooka-Gobara, Shiojiri, Japan. ${ }^{6}$ Hard Tissue Pathology Unit, Matsumoto Dental University Graduate School of Oral Medicine, 1780, Hirooka-Gobara, Shiojiri, Japan. ${ }^{7}$ Division of Microbiology and Oral Infection, Department of Molecular Microbiology and Immunology, Nagasaki University Graduate School of Biomedical Sciences, Nagasaki, Japan.
\end{abstract}

Received: 2 October 2014 Accepted: 9 January 2015

Published: 17 January 2015

\section{References}

1. Holt SC, Ebersole JL. Porphyromonas gingivalis, Treponema denticola, and Tannerella forsythia: the "red complex", a prototype polybacterial pathogenic consortium in periodontitis. Periodontol 2000. 2005:38:72-122.

2. Duran-Pinedo AE, Nishikawa K, Duncan MJ. The RprY response regulator of Porphyromonas gingivalis. Mol Microbiol. 2007;64(4):1061-74.

3. Krishnan K, Duncan MJ. Role of sodium in the RprY-dependent stress response in Porphyromonas gingivalis. PLoS One. 2013;8(5):e63180.

4. Diaz PI, Slakeski N, Reynolds EC, Morona R, Rogers AH, Kolenbrander PE. Role of oxyR in the oral anaerobe Porphyromonas gingivalis. J Bacteriol. 2006:188(7):2454-62.

5. Socransky SS, Haffajee AD. Dental biofilms: difficult therapeutic targets. Periodontol 2000. 2002;28:12-55.

6. Kolenbrander PE. Oral microbial communities: biofilms, interactions, and genetic systems. Annu Rev Microbiol. 2000;54:413-37.

7. Naito M, Hirakawa H, Yamashita A, Ohara N, Shoji M, Yukitake H, et al. Determination of the genome sequence of Porphyromonas gingivalis strain ATCC 33277 and genomic comparison with strain W83 revealed extensive genome rearrangements in $P$. gingivalis. DNA Res. 2008;15(4):215-25.

8. Kikuchi $Y$, Ohara N, Ueda O, Hirai K, Shibata Y, Nakayama K, et al. Porphyromonas gingivalis mutant defective in a putative extracytoplasmic function sigma factor shows a mutator phenotype. Oral Microbiol Immunol. 2009;24(5):377-83.

9. Dou Y, Osbourne D, McKenzie R, Fletcher HM. Involvement of extracytoplasmic function sigma factors in virulence regulation in Porphyromonas gingivalis W83. FEMS Microbiol Lett. 2010;312(1):24-32.

10. Yanamandra SS, Sarrafee SS, Anaya-Bergman C, Jones K, Lewis JP. Role of the Porphyromonas gingivalis extracytoplasmic function sigma factor, SigH. Mol Oral Microbiol. 2012:27(3):202-19.

11. Nakayama K, Kadowaki T, Okamoto K, Yamamoto K. Construction and characterization of arginine-specific cysteine proteinase (Arg-gingipain)deficient mutants of Porphyromonas gingivalis. Evidence for significant contribution of Arg-gingipain to virulence. J Biol Chem. 1995;270 (40):23619-26.

12. Ueshima J, Shoji M, Ratnayake DB, Abe K, Yoshida S, Yamamoto K, et al. Purification, gene cloning, gene expression, and mutants of Dps from the obligate anaerobe Porphyromonas gingivalis. Infect Immun. 2003;71(3):1170-8.

13. Nakayama K. Rapid viability loss on exposure to air in a superoxide dismutase-deficient mutant of Porphyromonas gingivalis. J Bacteriol. 1994;176(7):1939-43.

14. Gardner RG, Russell JB, Wilson DB, Wang GR, Shoemaker NB. Use of a modified Bacteroides-Prevotella shuttle vector to transfer a reconstructed $\beta$-1,4-D-endoglucanase gene into Bacteroides uniformis and Prevotella ruminicola B 4. Appl Environ Microbiol. 1996;62(1):196-202.

15. Saito Y, Fujii R, Nakagawa Kl, Kuramitsu HK, Okuda K, Ishihara K. Stimulation of Fusobacterium nucleatum biofilm formation by Porphyromonas gingivalis. Oral Microbiol Immunol. 2008;23(1):1-6.

16. Raivio TL. Envelope stress responses and Gram-negative bacterial pathogenesis. Mol Microbiol. 2005;56(5):1119-28.

17. Jordan S, Hutchings Ml, Mascher T. Cell envelope stress response in Grampositive bacteria. FEMS Microbiol Rev. 2008;32(1):107-46.

18. Bashyam MD, Hasnain SE. The extracytoplasmic function sigma factors: role in bacterial pathogenesis. Infect Genet Evol. 2004;4(4):301-8.

19. Mascher T, Helmann JD, Unden G. Stimulus perception in bacterial signaltransducing histidine kinases. Microbiol Mol Biol Rev. 2006;70(4):910-38.

20. Francez-Charlot A, Frunzke J, Reichen C, Ebneter JZ, Gourion B, Vorholt JA Sigma factor mimicry involved in regulation of general stress response. Proc Natl Acad Sci USA. 2009;106(9):3467-72.

21. Bordi C, de Bentzmann S. Hacking into bacterial biofilms: a new therapeutic challenge. Ann Intensive Care. 2011;1(1):19.

22. Luo Y, Asai K, Sadaie Y, Helmann JD. Transcriptomic and phenotypic characterization of a Bacillus subtilis strain without extracytoplasmic function sigma factors. J Bacteriol. 2010;192(21):5736-45.

23. Lin $\mathrm{X}, \mathrm{Wu} \mathrm{J}$, Xie H. Porphyromonas gingivalis minor fimbriae are required for cell-cell interactions. Infect Immun. 2006;74(10):6011-5.

24. Flemming $\mathrm{HC}$, Wingender J. The biofilm matrix. Nat Rev Microbiol. 2010;8(9):623-33.

25. Ali Mohammed MM, Nerland AH, Al-Haroni M, Bakken V. Characterization of extracellular polymeric matrix, and treatment of Fusobacterium nucleatum and Porphyromonas gingivalis biofilms with DNase I and proteinase K. J Oral Microbiol. 2013;5:20015.

26. Kuboniwa M, Amano A, Hashino E, Yamamoto Y, Inaba H, Hamada N, et al. Distinct roles of long/short fimbriae and gingipains in homotypic biofilm development by Porphyromonas gingivalis. BMC Microbiol. 2009;9:105.

27. Yamamoto R, Noiri Y, Yamaguchi M, Asahi Y, Maezono H, Kuboniwa M, et al. The sinR ortholog PGN_0088 encodes a transcriptional regulator that inhibits polysaccharide synthesis in Porphyromonas gingivalis ATCC 33277 biofilms. PLoS One. 2013;8(2):e56017.

28. Holt SC, Kesavalu L, Walker S, Genco CA. Virulence factors of Porphyromonas gingivalis. Periodontol 2000. 1999;20:168-238.

29. Lamont RJ, Jenkinson HF. Life below the gum line: pathogenic mechanisms of Porphyromonas gingivalis. Microbiol Mol Biol Rev. 1998;62(4):1244-63.

doi:10.1186/1472-6831-15-4

Cite this article as: Onozawa et al:: Role of extracytoplasmic function sigma factors in biofilm formation of Porphyromonas gingivalis. BMC Ora Health 2015 15:4.

\section{Submit your next manuscript to BioMed Central and take full advantage of:}

- Convenient online submission

- Thorough peer review

- No space constraints or color figure charges

- Immediate publication on acceptance

- Inclusion in PubMed, CAS, Scopus and Google Scholar

- Research which is freely available for redistribution 